\title{
Coping with Bleeding Disorders
}

\section{Tony Brown*}

Hemophilia Treatment Center, University of South Florida, USA

*Corresponding author: Tony Brown, Hemophilia Treatment Center, University of South Florida, USA

\begin{abstract}
Living with a bleeding disorder is something that very few live within the United States. In fact, hemophilia occurs in only approximately 1 in 5,000 live births. There are about 20,000 people with hemophilia A in the US. Hemophilia B is four times less common than hemophilia A. von Willebrand Disease is much more common than both hemophilia $A$ \& $B$ which is believed to effect $1 \%$ of the population. Von Willebrand disease is estimated to affect 1 in 100 to 10,000 individuals. The 3 common bleeding disorders discussed is hemophilia A \& B as well as von Willebrand Disease. Living with a bleeding disorder is a challenge for most patients. The topic of discussion is the ability for patients to cope with pain. The pain coping is ranged from $0-5$ with 0 being "not applicable", 1 being "poor" and 5 being "very good". The subjects were gathered during a yearly comprehensive hemophilia clinic visit at the University of South Florida. The method used to gather participants was using a random design. This study used the data for a true experimental design which requires random assignment into a control group. The study is a blind design where the patients used in the study were not identified by location, name, sex or other identifiable diagnosis. The information used in data entry was the bleeding disorder type and age. We discussed how bleeding disorder types and age affect the ability of a patient to cope with pain. We will also look at the effects of having a bleeding disorder on the patient.
\end{abstract}

\section{Mission}

The mission of this paper is to discover the coping of patients living with hemophilia. This patient will look into possible outcome of those that have a bleeding disorder and how it affects their day to day life. We will look at possible disabilities associated to the bleeding disorder. While looking into the bleeding disorder we will see if there is a correlation between possible disabilities and stress/depression. The bleeding disorder discussed in this paper is hemophilia A, hemophilia B as well as various types of von Willebrand Disease.

\section{Hemophilia}

There are 13 numerals but only 12 factors. Factor VI was subsequently found to be part of another factor. The liver must be able to use Vitamin $\mathrm{K}$ to produce Factors II, VII, IX, and X. Dietary vitamin K is widely available from plant and animal sources. Hemophilia is a bleeding disorder where the blood does not clot properly. The most common hemophilia is A and B, which is also called factor VIII (antihemophilic factor) and factor IX (plasma thromboplastin component) deficiency. In a person with a bleeding disorder, the first two stages of homeostasis function normally the blood vessels tighten, an undeveloped platelet plug is formed, and bleeding may stop or slow down [1]. However, since one of the clotting factors is reduced or absent, a fully developed fibrin clot is not created; instead, a soft, ineffective clot is formed [1]. The soft or weakened platelet in a hemophiliac patient breaks down which causes the bleeding to continue.

The most common bleeding disorder, hemophilia, is an X-Linked genetic disorder characterized by a deficiency or absence of factor VIII or factor IX. Hemophilia is not a very common deficiency which is the reason that it account for 1 in every 5032 males [1]. It is believed that about 400 babies of all racial and ethnicities are born with hemophilia each year in the United States. Babies born with a factor deficiency are $4 \mathrm{x}$ more likely to develop factor VIII than IX [1].

Hemophilia is carried on an abnormal X-linked chromosome. A female hemophilia carrier is when the hemophilia gene is on one of her $2 \mathrm{X}$ chromosomes. It is often discovered that a hemophilia carrier exhibits symptoms. Having one $X$ may not cause the female to have hemophilia if the normal gene on the other $X$ chromosome compensates [1]. Males do not transmit the

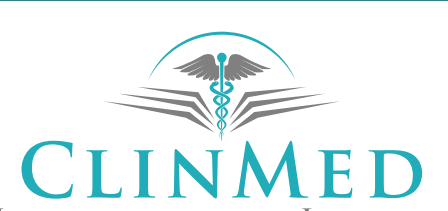

INTERNATIONAL LIBRARY
Citation: Brown T (2020) Coping with Bleeding Disorders. Int J Blood Res Disord 7:058. doi. org/10.23937/2469-5696/1410058

Accepted: October 12, 2020: Published: October 14, 2020

Copyright: (c) 2020 Brown T. This is an open-access article distributed under the terms of the Creative Commons Attribution License, which permits unrestricted use, distribution, and reproduction in any medium, provided the original author and source are credited. 
disorder to their sons but all of their daughters may become carriers of the deficiency. One third of hemophilia cases there is no family history of the deficiency. When this rare occurrence happens it is believed to be a result of a recent genetic mutation.

Due to the bleeding disorder many patients develop the bleeds that affect their day to day lives. Many of the patients exhibit symptoms that include target joints and other issues that may have been a result of the frequent bleeds. Some of the target includes but not limited to the knees, ankles, shoulders, elbow, hip, wrist etc. to better control the frequency of the bleeds many patient treat with antihemophilic medication.

\section{Von Willebrand Disease}

Von Willebrand Factor is a large and complex plasma glycoprotein that is essential for normal homeostasis. Von Willebrand also carries the coagulation factor VIII to the area of clot formation. Von Willebrand disease is defined as a bleeding disorder that is chiefly attributable to decreased levels of VWF activity [2]. This is often, but not always, attributable to a defect in the VWF gene. Bleeding histories can be individualized and the disease characteristics can take time to develop. It is believed that there is an overlap between symptoms suffered by people with VWD and the normal population [2].

Levels of von Willebrand and FVIII can rise in the first trimester of pregnancy and increase with gestational stage reaching two to three times those of the non-pregnant state at term. Levels often times fall after delivery, returning to normal non-pregnant levels within a few weeks [2]. Because of this possible occurrence, women are just as likely to develop von Willebrand Disease as their male counterparts. Equal occurrence of the deficiency is not something expected with hemophilia. However, women are more likely to experience symptoms of VWD because of the increased bleeding it causes during their menstrual periods, during pregnancy, and after childbirth [2]. Von Willebrand Disease is prevalent in $1 \%$ of the general population. The prevalence in von Willebrand Disease exceeds that of both factor VIII and factor IX deficiency. Hemophilia occurs in one and every 5032 males, von Willebrand disease occurs in one of every 125 people in the United States [2].

\section{Disabilities}

Now that we understand the common types of bleeding disorders, to truly understand the impact of the bleeding disorder on an individual we must understand how society defines it. Definition of disability is something that is not universally defined. Disability has its own definition depending on its region and how the individual define the word. According to Brandt and Pope, disability is limitation in performing certain roles and tasks that society expects an individual to perform [3]. Disability is the appearance of the gap between demands of the environment and individual's capabilities [3].
In actuality disability are the social restrictions in the world around the individual, not being a person with a disabling condition. The individual may have some form of disabling rather physical or mental health but, the experience of disability is created through the constant interactions with the social and physical world. Lots of times disability is perceived by the outside world resulting of the discrimination, stigmatization, prejudice and forced independence on relatives and healthcare individuals [3].

Many of the people with hemophilia get by fine as far as maintaining a healthy lifestyle as well as relationships. The challenges that causes disability to become an issue with many is Ableism. Ableism is a term used to describe discriminatory actions in the favor of the able-bodied [4]. Many of this can occur because of stereotypes associated to having a bleeding disorder or the visible physical limitations.

\section{Methodology}

\section{Sampling}

The samples used in this particular study were the evaluating of the adults patients with a bleeding disorder over the age of 18 . The sampling was based on sample size given from gathered data of a population of 45 . The population includes all those who met the bleeding disorder criteria as well as physical limitations.

The characteristics of the sampling include 45 patients between the ages of 18 and 80 -years-old. The sampling summary consists of information generated from the new and established patients that visiting for a comprehensive bleeding disorder clinic at the University of South Florida. All participating must be adult patient minimum of 18 and diagnosed with a bleeding disorder such as hemophilia or von Willebrand Disease. The race categories includes white, black, Hispanic and other. Due to the limited sample size and the need to keep identity limited, the patient's race was not asked. The populated of patients volunteered in the study were from various locations in Florida.

\section{Inclusion and exclusion}

The inclusion criteria for the study were those who met the criteria for the Hemophilia Treatment Center. The eligibility criteria include males and females diagnosed with either Factor 7, 8, 9 as well as von Willebrand Disease. The study includes the data collected for the adult patients over 18 seen during comprehensive clinics from April 2017 until August 2019. The sampling did not include those who aged out of the program prior to April 2017 or after August 2019.

\section{Procedure}

During the first month working with clients with chronic bleeding disorders the Medical Social Worker at the University of South Florida discovered that many 
of the patient's exhibit signs of discomfort and/or anxiety. The social worker decided to look into the reasons for the patient's anxiety and extreme discomfort at the time of the comprehensive visits. A coping assessment was created to discover the levels of for patients with bleeding disorders. The coping assessment was to discover the patient's ability to divert attention when in pain, understanding of the pain, and outlook on normal day to day. The patient data was collected to see if bleeding disorders have a high level of stress and anxiety.

\section{Results}

A non-probability sampling was used to because of the convenience of gathering the information. A simple random sampling was also used to create an equal chance of the bleeding disorder patients to be selected. The other reason for using this particular design was because the group was used throughout the study due to its simplicity.

The results shows that patients with hemophilia $(A$, B) have a pain intensity of 2.7 while patients with von Willebrand Disease has a score of 4.7. The pain intensity demonstrates how patients interpret pain. What was discovered also shows that the patient's scored high when it comes to coping with the pain without medication. The average score for patients with von Willebrand Disease and Hemophilia was 4.4.

The patient's negative thoughts were as expected. The patients with hemophilia had negative thoughts with pain nearly every day and or more than average. The patient's with von Willebrand disease scored either never or not often when answering about the negative thoughts when in pain.

The patients were also asked about the use of pain medication to help with diverting pain. The patients with hemophilia were $2 x$ more likely to score of taking pain medication more than average or nearly every day. The patients with von Willebrand disease scored not often or never when it pertains to taking pain medications.

The last discovery showed that patients typically maintain happiness and feeling hopeful with having bleeding disorders. The few that scored different were those with hemophilia rather than von Willebrand disease. The patient's on average scored 2.5 which is what was expected. The 5 patients that scored high on the assessments brought the overall score up. Without the 5 patient's the average score was 1.2 which is between not often and never.

\section{Limitations}

The overall discovery in the study showed those patients with pain more often than average have a negative thought process about self and bleeding disorder. This is something that was expected when it pertains to bleeding disorder. The patients that experience pain often have negative thoughts and some depression. Depression is something that is felt because of the inability to cope with the pain. When pain is something experience nearly every day it is hard to think of something else. One major flaw within the study is not asking about support and current use of self-care.

Had self-care been asked this would show which patient's need extra attention on finding ways to divert pain. Many times patients do not understand the things that can be done to relieve pain. Having lots of support in the area also can help with keeping positive. The additional information could also further understand the level of stress and negative thoughts about self.

The biggest thing found in this study is that patients on average were happy with themselves. Aside from the few patients the majority of the patients kept positive and feels happy about themselves. The majority of the patients find the beauty in things despite having pain. The patient's on average does well with diverting attention when in pain. This was something positive discovered because, despite having pain more often than normal the patients find the beauty in things and are not negative on themselves. Future research will look into the reason the few patients that have pain negative thoughts and feeling of hopelessness.

\section{References}

1. Regina B Butler (2012) Introduction to Bleeding Disorders. National Hemophilia Foundation 1-8.

2. Mike A Laffan, Will Lester, James S O'Donnell, Andrew Will, Robert Campbell Tait, et al. (2014) The diagnosis and management of von Willebrand disease: A United Kingdom hemophilia centre doctors organization guideline approved by the British committee for standards in hematology. Br J Hematol 167: 453-465.

3. Lutz BJ, Bowers BJ (2007) Understanding how Disability is Defined and Conceptualized in the Literature. In $A E$ Dell Orto, PW Power, Psychological and Social Impact of IIIness and Disability ( $5^{\text {th }}$ edn). New York: Springer Publishing Company.

4. Alshammari S (2016) Disability, coping, and identity. Journal of Literary \& Cultural Disability Studies 10: 361-367. 